\title{
Identification of a novel pathogenic $C O L 4 A 3$ gene mutation in a Chinese family with autosomal dominant Alport syndrome: A case report
}

\author{
DA-AN NIE $^{1^{*}}$, CHAO-RUI XIA $^{1 *}$, KE-CHENG HUANG ${ }^{2}$, JIE LIU ${ }^{1}$, \\ TING GAN $^{1}$, CHENG WEN ${ }^{3}$ and ZHI-PENG ZENG ${ }^{4}$ \\ ${ }^{1}$ Department of Cardiology, Union Hospital; ${ }^{2}$ Department of Obstetrics and Gynecology, Tongji Hospital, \\ Tongji Medical College, Huazhong University of Science and Technology, Wuhan, Hubei 430030; \\ ${ }^{3}$ Department of Endocrinology, Xiaogan First People's Hospital, Xiaogan, Hubei 432000; \\ ${ }^{4}$ Department of Rheumatology and Immunology, Tongji Hospital, Tongji Medical College, \\ Huazhong University of Science and Technology, Wuhan, Hubei 430030, P.R. China
}

Received December 21, 2020; Accepted July 28, 2021

DOI: $10.3892 /$ br.2021.1466

\begin{abstract}
Alport syndrome (AS) is a genetic disease with various manifestations, including hematuria, proteinuria, impaired renal function and potential ocular or auditory abnormalities. Mutations in the collagen type IV $\alpha 3$ chain (COL4A3), collagen type IV $\alpha 4$ chain and collagen type IV $\alpha 5$ chain genes encoding the $\alpha 3, \alpha 4$ and $\alpha 5$ chains of type IV collagen may undermine glomerular basement membrane (GBM) integrity and cause persistent renal deterioration. In the present study, the case of a Chinese family diagnosed with AS was examined. Pedigree investigations and whole exome sequencing (WES) revealed the presence of two heterozygous mutations (c.2603G >A; p.G868E, and c.583G>A; p.G195S) in the COL4A3 gene. p.G868E was identified as the 'culprit' mutation, whereas p.G195S was identified as an 'auxiliary' mutation for AS with regards to the manifestations observed in the patients carrying each of the gene mutations. In conclusion, these findings suggested that c. $2603 \mathrm{G}>\mathrm{A}$ may be a novel overt pathogenic mutation site for autosomal dominant AS. In addition, WES may be effective for the early diagnosis and medical intervention of AS, and may be widely used for AS prognosis prediction and pre-implantation genetic diagnosis.
\end{abstract}

Correspondence to: Dr Zhi-Peng Zeng, Department of Rheumatology and Immunology, Tongji Hospital, Tongji Medical College, Huazhong University of Science and Technology, 1095 Jiefang Avenue, Wuhan, Hubei 430030, P.R. China E-mail: 512527720@qq.com

*Contributed equally

Key words: Alport syndrome, end stage renal disease, type IV collagen, COL4A3 gene, whole exome sequencing

\section{Introduction}

Alport syndrome (AS) is a hereditary disease characterized by hematuria, proteinuria and chronic progressive failure of renal function, as well as potential ocular abnormalities and sensorineural deafness $(1,2)$. The basic pathological mechanism of AS is glomerular basement membrane (GBM) abnormalities, due to pathogenic mutations in the genes encoding the $\alpha 3, \alpha 4$ and $\alpha 5$ chains of type IV collagen $(2,3)$. AS is generally divided into three inherited types. The most common type is X-linked dominant hereditary type AS (XLAS), which accounts for $>80 \%$ of AS cases, and involves a mutation in the gene encoding the $\alpha 5$ chain (COL4A5) located at chromosome $\mathrm{Xq} 2$. Meanwhile, autosomal recessive AS (ARAS), which accounts for $15 \%$ of AS cases, and rare autosomal dominant AS (ADAS), which accounts for 5\% of AS cases, are induced by mutations in the collagen type IV $\alpha 3$ chain (COL4A3) and collagen type IV $\alpha 4$ chain (COL4A4) genes located at autosomal chromosome 2q36.3 (4,5).

AS had been historically diagnosed based on the clinical manifestations of hematuria, proteinuria and renal dysfunction; however, the use of renal biopsy with light microscopy (LM) and electron microscopy (EM), or immunohistochemical staining has become more popular in the past five decades (4). Ear and eye tests, alongside pedigree analysis have proven helpful for improving the accuracy of an AS diagnosis (4,6). However, missed diagnoses are still common in AS, and early intervention is crucial to delay disease progression; therefore, more precise diagnostic tools are required (4). Advances in genetic diagnostic technologies have largely made up for the shortcomings of traditional tools and have revealed that genotype defects are not linearly correlated with the various clinical phenotypes $(2,6)$. In particular, patients with ADAS with heterozygous mutations are clinically characterized by slower end-stage renal disease (ESRD) progression with less typical extra-renal manifestations compared with ARAS, in which gene mutations usually occur in both alleles (6). Thus, the incidence of missed diagnoses for ADAS could potentially be higher than previously suggested (7), 
which could be sufficiently treated by early administration of angiotensin-converting-enzyme inhibitor (ACEi) $(4,6,8)$. Therefore, the introduction of whole-exome sequencing (WES) analysis can markedly improve the diagnostic and prognostic accuracy for AS $(3,6)$.

In the present study, a proposed AS proband was identified based on the patients' clinical manifestations and was further confirmed by pathological examination. WES was recommended for the proband to determine the exact mutation sites. Two novel pathogenic COL4A3 mutations (c.2603G $>$ A; p.G868E, and c.583G $>$ A; p.G195S) were identified. The results of the present study enrich the pool of mutations associated with ADAS, whilst showing that WES may be a powerful tool for AS diagnosis and mutation characterization, as well as for assisting with precise medical intervention, prognosis prediction and a pre-implantation genetic diagnosis (6).

\section{Case report}

The present study was approved by the Ethics Committee of Tongji Hospital, Tongji Medical College, Huazhong University of Science and Technology (Wuhan, China). Written informed consent was obtained from each patient, or from the parents of any patients $<18$ years old, for participation in the study. The proband (III.2) was a 13-year-old male patient who was admitted to the pediatric nephrology department of Tongji Hospital after presenting with persistent hematuria for $>8$ years. The proband (III.2) and 9 members of the family (6 men and 4 women) clinically suspected of AS were recruited. All patients underwent blood and urine routine examination, as well as liver and kidney function evaluations. Eyesight and visual filed inspection, hearing tests and vestibular function tests were also performed, where necessary.

The family's clinical history was examined carefully for each case through the reconstruction of the pedigree for at least three generations (Fig. 1A). The renal biopsy of the proband (III.2) and his older sister (III.1) were examined in the nephrology laboratory of Tongji Hospital. The proband fulfilled three of the AS clinical diagnostic criteria: The family history of renal failure, hematuria and poor vision (6). The hearing test and vestibular function test were normal. The eye test revealed retinal detachment in the left eye. At the age of 13 years, urine analysis revealed proteinuria $(2.8 \mathrm{~g} / 24 \mathrm{~h})$, so a renal biopsy was performed. The abnormal GBM was identified by EM, which was compatible with the typical ultrastructural abnormalities characteristic of thin basement membrane disease. Antiproteinuric treatment was reinforced with ACEi and tacrolimus.

\section{Materials and methods}

Whole blood genomic DNA extraction. Fresh venous blood (3 ml) was obtained from patients of the pedigree for genomic DNA extraction, following the manufacturer's instructions (QIAamp; Qiagen AB).

WES and bioinformatics analysis. WES was performed by Beijing Genomics Institution in Shenzhen, China. The experimental procedures were performed as described previously (9). Briefly, the concentration and purification of the proband's DNA (OD260/280>1.8) was detected using a NanoDrop ${ }^{\mathrm{TM}} 2000$ spectrophotometer (Thermo Fisher Scientific, Inc.), and a $1 \mu \mathrm{g}$ sample was used for DNA library construction. Genomic DNA was randomly broken into 150-250-bp-long fragments using a Covaris S2 Sonicator (Covaris). End repairing, A-tailing and adapter ligation of fragments were performed in a single tube (total $110 \mu \mathrm{l}$ ) for $15 \mathrm{~min}$ at $22^{\circ} \mathrm{C}$ (iGeneTech, Inc.), followed by a purification with $88 \mu 1$ Agencourt Ampure XP beads (Beckman Coulter, Inc.), and collected in $20 \mu 1$ nuclease-free water. After pre-PCR amplification $\left(9^{\circ} \mathrm{C}\right.$ for $3 \mathrm{~min}$; followed by 8 cycles of $98^{\circ} \mathrm{C}$ for $20 \mathrm{sec}, 60^{\circ} \mathrm{C}$ for $15 \mathrm{sec}$ and $72^{\circ} \mathrm{C}$ for $30 \mathrm{sec}$; with a final extension step at $72^{\circ} \mathrm{C}$ for $10 \mathrm{~min}$; and held at $4^{\circ} \mathrm{C}$ ) using a Library Prep kit (iGeneTech, Inc.), the product was purified using Agencourt Ampure XP beads and quantified using a Qubit dsDNA BR assay kit (Thermo Fisher Scientific, Inc.).

The purified PCR products were hybridized with a human gene chip for 68-72 h using an MGIEasy Exome Capture V4 Probe Set (MGI Tech Co., Inc.). The target fragments were captured by the Cap beads (iGeneTech, Inc.), and then underwent further capture post-PCR $\left(95^{\circ} \mathrm{C}\right.$ for $3 \mathrm{~min}$; followed by 8 cycles of $98^{\circ} \mathrm{C}$ for $20 \mathrm{sec}, 60^{\circ} \mathrm{C}$ for $15 \mathrm{sec}$ and $72^{\circ} \mathrm{C}$ for $30 \mathrm{sec}$; with a final extension step at $72^{\circ} \mathrm{C}$ for $10 \mathrm{~min}$; and held at $4^{\circ} \mathrm{C}$ ) using a TargetSeq One kit (iGeneTech, Inc.). The PCR products were purified using Agencourt Ampure XP beads, qualified using the Qubit ssDNA kit (Thermo Fisher Scientific, Inc.) and amplified to make DNA nanoballs (10), which consist of $>300$ copies of one molecule. Pair-end 100 bases reads were generated using the combinatorial Probe-Anchor Synthesis-based BGISEQ-500 platform (BGI) as described previously (10).

The obtained sequences were compared to those of the human reference genome (hg19) using the Burrows-Wheeler Aligner software (version 0.7.15) to acquire valid sequences, and potential pathogenic variants [by comparing with dbSNP, 1000 Genomes Project, Online Mendelian Inheritance in Man (OMIM) and ClinVar]. The function of variants was predicted using SIFT version 6.1 (sift.bii.a-star.edu.sg) and PolyPhen-2 (genetics.bwh.harvard.edu/pph2). The conservation of amino acid sequences of $C O L 4 A 3$ gene variants was analyzed using the NCBI database (ncbi.nlm.nih.gov/protein).

Sanger sequencing verification of COL4A3 gene mutation. According to the gene identification site, fragments were amplified by PCR with the following thermocycling conditions: $95^{\circ} \mathrm{C}$ for $15 \mathrm{~min}$; followed by 30 cycles of $94^{\circ} \mathrm{C}$ for $30 \mathrm{sec}$, $53^{\circ} \mathrm{C}$ for $30 \mathrm{sec}$ and $72^{\circ} \mathrm{C}$ for $30 \mathrm{sec}$; with a final extension step of $72^{\circ} \mathrm{C}$ for $10 \mathrm{~min}$. The sequences of the primers were: c.583G $>$ A forward, 5'-AGGGAGGCGGAGGGTAC-3' and reverse, 5'-ATGGCCCCTGGTTTCTTAC-3'; and c.2603G>A forward, 5'-AGGGAGGCGGAGGGTAC-3' and reverse, 5'-ATGGCCCCTGGTTTCTTAC-3'. PCR products were sequenced by Sanger sequencing (ABI 3730XL sequencer; Thermo Fisher Scientific, Inc.) and the Sanger sequencing peak map and results were analyzed on DNASTAR Lasergene version 13.0 (DNASTAR, Inc.).

\section{Results}

The clinical investigation of the pedigree suggested the presence of hereditary ADAS within the family (Fig. 1A). The 
A

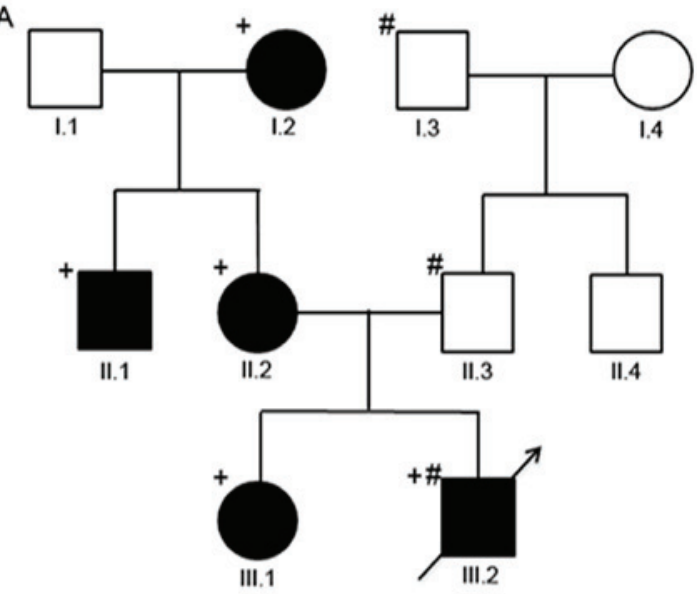

B

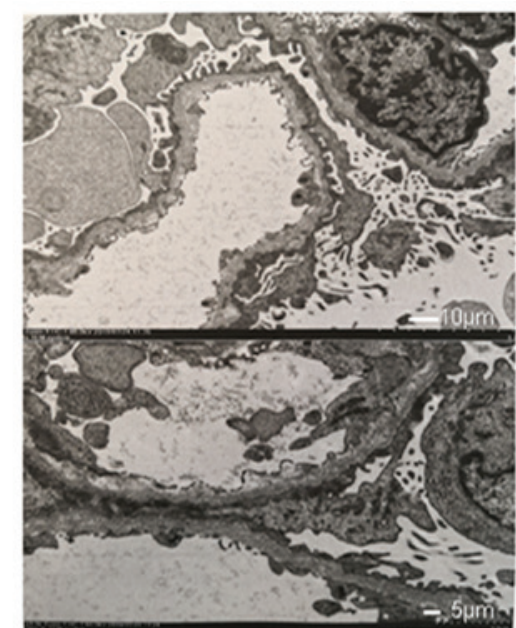

C

WT

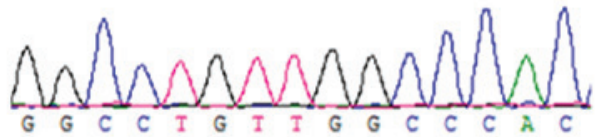

Mutation

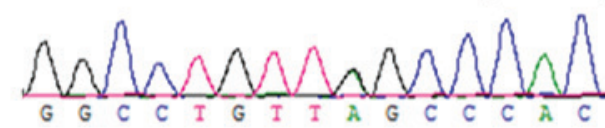

D

$\begin{array}{ll}\text { Mutation } & \text { GFPGPVSPPGPPGFF } \\ \text { H.sapiens } & \text { GFPGPVGPPGPPGFF } \\ \text { P.troglodytes } & \text { GFPGPVSPPGPPGFF } \\ \text { M.mulatta } & \text { GFPGPVSPPGPPGFF } \\ \text { F.catus } & \text { GFPGPVGPPGPPGLF } \\ \text { M.musculus } & \text { GFPGPAGPPGPPGFF } \\ \text { G.gallus } & \text { GYPGETGPRGLPGTS } \\ \text { T.rubripes } & \text { GNPGPRGSPGNPGHG }\end{array}$

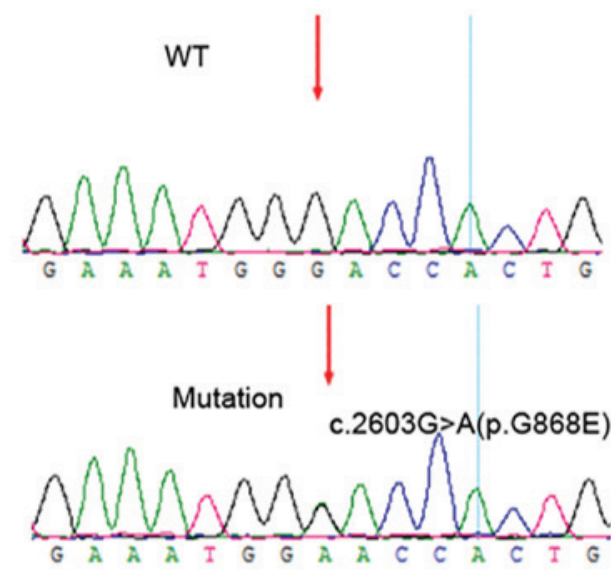

Mutation
H.sapiens
P. troglodytes
M.mulatta
F.catus
M.musculus
G.gallus
T.rubripes

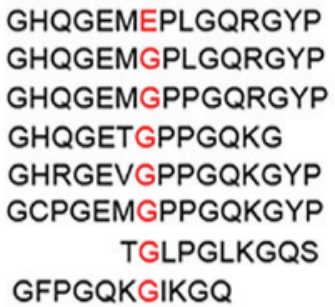

Figure 1. Findings of the pedigree analysis and COL $4 A 3$ gene mutations. (A) Pedigree analysis of the family. (B) Electron microscopy image of the proband's kidney biopsy. The octothorpe indicates p.583G $>$ A and the cross, c.2603G $>$ A. (C) Two heterozygous nucleic acid substitutions (c.583G $>$ A and c.2603G $>$ A) were screened in the COL4A3 gene from the proband's DNA sample. (D) The site is highly conserved amongst mammalians. Squares indicate men and circles women. The arrow indicates the proband. $C O L 4 A 3$, collagen type IV $\alpha 3$ chain; WT, wild-type.

proband was a 13-year-old boy, who presented with repeated gross hematuria aggravated by fever for 8 years, and whose left vision had been progressively decreasing for 2 years due to retinal detachment. Routine urine routine revealed the following abnormal results: Red blood cells (RBC), 3+; white blood cells, \pm ; urinary albumin, 1+; microscopic RBC per full field/high power; irregular RBC \%, 30.0\%; and 24 h urinary albumin, $2.8 \mathrm{~g}$. The levels of serum creatinine (Scr) and blood urea nitrogen (BUN) were normal. Renal biopsy showed partial glomerular sclerosis, mild mesangial cell hyperplasia and slightly increased mesangial matrix content without segmental necrosis and thrombosis under LM. No obvious abnormalities were identified by immunofluorescence. EM revealed that the capillary basement membrane was segmentally thinned, and the lowest thickness was $160 \mathrm{~nm}$ (physiological thickness is $\sim 300 \mathrm{~nm}$ ), and even some dense layers were torn and delaminated (Fig. 1B). These results suggested that the proband could be diagnosed with AS and recommended for genetic testing.

The pedigree investigation revealed hereditary ADAS (Table I). The proband's 15-year-old sister had abnormal urinalysis findings for 1 year, including the following: RBC, 3+; urinary albumin, 2+; $24 \mathrm{~h}$ urinary albumin, $500 \mathrm{mg}$. The levels of Scr $(65 \mu \mathrm{mol} / \mathrm{l})$ and BUN (4.52 mmol/l) were normal. Renal biopsy under LM revealed segmental mesangial cells and mild stromal hyperplasia with segmental endothelial proliferation. Her EM results were similar to those of her younger brother (data not shown). The sister had normal hearing and vision after examination. Thus, the sister could also be diagnosed with AS. In the maternal family, the grandmother had uremia, whereas 
Table I. Clinical and molecular characteristics of the recruited patients.

\begin{tabular}{|c|c|c|c|c|c|c|}
\hline Patient & Sex & Age & Hematuria & Proteinuria & Renal Function & Mutations \\
\hline I1 & M & 68 & No & No & Normal & Normal \\
\hline $\mathrm{I} 2$ & $\mathrm{~F}$ & 65 & Yes & $>3.5 \mathrm{~g} / 24 \mathrm{~h}$ & $\begin{array}{l}\text { Chronic renal } \\
\text { failure-dialysis }\end{array}$ & c. $2603 \mathrm{G}>\mathrm{A} ;$ p.G868E \\
\hline I3 & M & 70 & No & No & Normal & c.583G >A; p.G195S \\
\hline I4 & $\mathrm{F}$ & 67 & No & No & Normal & Normal \\
\hline II1 & M & 35 & Yes & $1.2 \mathrm{~g} / 24 \mathrm{~h}$ & Normal & c. $2603 \mathrm{G}>\mathrm{A} ;$ p.G868E \\
\hline II 2 & $\mathrm{~F}$ & 37 & Yes & No & Normal & c. $2603 \mathrm{G}>\mathrm{A} ;$ p.G868E \\
\hline II3 & M & 41 & No & No & Normal & c.583G>A; p.G195S \\
\hline II4 & M & 43 & No & No & Normal & Normal \\
\hline III1 & $\mathrm{F}$ & 15 & Yes & $0.5 \mathrm{~g} / 24 \mathrm{~h}$ & Normal & c. $2603 \mathrm{G}>\mathrm{A} ;$ p.G868E \\
\hline III2 & M & 13 & Yes & $2.8 \mathrm{~g} / 24 \mathrm{~h}$ & Normal & $\begin{array}{l}\text { c. } 2603 \mathrm{G}>\mathrm{A} ; \text { p.G868E } \\
+ \text { c. } 583 \mathrm{G}>\mathrm{A} ; \text { p.G195S }\end{array}$ \\
\hline
\end{tabular}

F, female; M, male.

the mother and uncle had chronic glomerulonephritis without ocular or auditory abnormalities. No significant abnormal manifestations were observed in the paternal members.

The proband's WES raw data output was $22.71 \mathrm{G}$, and the target area length was $58.68 \mathrm{Mb}$ with $99.92 \%$ coverage and an average sequencing depth of $82.93 \mathrm{X}$, which met the experimental design requirements. WES identified two mutations in the proband. One located in exon 10 of the COL4A3 gene (NM_000091.4) on chromosome 2, in which $\mathrm{G}$ in the $583 \mathrm{rd}$ position was changed to $\mathrm{A}$ (c.583G $>\mathrm{A}$ ), leading to the $\mathrm{GGC} \rightarrow \mathrm{AGC}$ codon change and the amino acid change from Gly to Ser (p.G195S; Fig. 1C). The other mutation was the change from $G$ to $A$ in the 2,603rd position in exon 32 of the same gene, resulting in the GGA $\rightarrow$ GAA codon change and 868th amino acid changing from Gly to Glu(p.G868E; Fig. 1D). These two mutations were extremely rare and had not been detected before in databases such as dbSNP, 1000 Genomes Project, OMIM, Human Gene Mutation Database and ClinVar.

All members of the pedigree underwent Sanger sequencing for the COL4A3 gene. The c. $2603 \mathrm{G}>\mathrm{A}$ mutation was found in four family members who presented with various AS symptoms, including the mother, uncle and grandmother of the maternal family and the proband's older sister (Table I). The c.583G $>$ A mutation was found in the proband's father and grandfather, who had normal urinalysis results, whereas the grandfather-in-law, who also had normal urinalysis readings, did not carry any mutations (Table I). Therefore, this pedigree could be diagnosed with hereditary ADAS, with the causal mutation site of the COL $4 A 3$ gene being c.2603 G>A (p.G868E).

Protein function prediction using SIFT and PolyPhen-2 software revealed both p.G195S and p.G868E to be pathogenic mutations. In addition, conserved sequence analysis showed the Gly at the 195th and 868th position in the COL4A3 gene was highly conserved in several species (Fig. 1D), suggesting that these positions could have fundamental influence over the structure of the three chains of type IV collagen and the biological function of the organism. According to the ACMG standards (11), the p.G868E variant is classified as a pathogenic variant and p.G195S is classified as likely pathogenic.

\section{Discussion}

AS is typically characterized by hematuria, proteinuria and progressive chronic renal failure, and may be accompanied by ocular and auditory abnormalities in certain patients, based on their gene mutations and disease progression $(1,2)$. The pathology of AS involves GBM abnormalities due to developmental defects in the $\alpha 3, \alpha 4$ and $\alpha 5$ chains of type IV collagen $(2,4)$. GBM abnormalities underlie the various manifestations of patients with AS, with gene mutations being the primary culprits of the GBM abnormalities (4). Of note, a patient's diagnosis and genotypes are not entirely deterministic, as several parameters are involved in AS progression $(4,6)$. However, early ACEi treatment is particularly effective in preventing disease progression to $\operatorname{ESRD}(2,4)$. Thus, early precise diagnosis and intervention are crucial for patient prognosis, and next-generation sequencing (NGS) testing may be a useful tool in the selection of a therapeutic strategy for AS (6,7).

The clinical manifestations of AS in patients primarily depends on their gene type classification, that is XLAS, ARAS and ADAS (4,5). Each type has different presentation and prognosis, since the outcomes of AS are based on various factors, such as mutation site, sex, age, family history, course, complications and treatment $(2,12)$. With regard to ADAS, the manifestations typically emerge during the childhood or teenage years, and will ultimately deteriorate gradually into ESRD if no medical interventions are performed; however, these patients have less auditory or ocular abnormalities (6). In the present study, the proband presented with gross hematuria for 8 years, following laboratory examinations and further confirmation using a kidney biopsy. The proband's 15 -year-old sister was also diagnosed with AS following laboratory examinations and significant findings in a pathological biopsy, which deemed pedigree investigation necessary. As suspected, three 
maternal members had AS, presenting with heterogenous symptoms; the middle-aged mother and uncle had chronic glomerulonephritis, whereas the disease in the grandmother had progressed into ESRD. The proband had ocular abnormalities, but no such abnormalities were observed in any of the other family members. Imafuku et al (13) reported that only 2 out of the 14 patients with ADAS exhibited loss of hearing $(14.2 \%)$ and none had ocular changes. These results imply that the clinical manifestations of AS are varied, from isolated hematuria, proteinuria, mild renal insufficiency to ESRD.

All patients with AS in the maternal family were found to possess a single p.G868E mutation that had given rise to various AS manifestations, which largely depended on the age of the three maternal members. These findings were consistent with those of a previous report of ADAS, where hematuria first emerged during childhood, likely as the only symptom, and proteinuria then gradually became prominent during puberty, rendering the ultimate progression to ESRD inevitable, given the persistent deterioration of renal function and lack of effective medical intervention for decades (14). Therefore, p.G868E is a pathogenic mutation and the patients carrying it can be diagnosed with ADAS.

However, the proband's clinical examination results were not in line with the typical course of ADAS development. The patient first presented with hematuria at the age of 5 years, which is not rare amongst pediatric patients with ADAS; however, the patient gradually presented with proteinuria and the aggravation of hematuria, which are not common findings amongst teenagers with ADAS. In particular, the age of onset preceded that of his older sister (who had abnormal hematuria and proteinuria for only 1 year) by several years. These findings suggested that other auxiliary mutations may have been implicated in the proband's disease course.

The proband had two mutations on the COL $4 A 3$ gene, whereas his older sister had only one mutation, which she had inherited from the mother. The aforementioned p.G868E mutation was derived from the maternal family, which laid the foundation for ADAS development. The other p.G195S mutation originated from the paternal family. The latter was not deterministic for AS onset, which was demonstrated by the fact that the carriers, including the father and grandfather, did not exhibit any abnormal AS manifestations. Thus, it is inferred that p.G195S is a pathogenic mutation that can potentially contribute to AS acceleration, but is not sufficient to induce AS on its own. Type IV collagen is the primary component of GBM, with embryonic $\alpha 1 \alpha 1 \alpha 2$ being replaced with the mature $\alpha 3 \alpha 4 \alpha 5$ heterotrimer in childhood to ensure integral structure and full function $(1,15)$.

The $\alpha 3$ chain encoded by the COL $4 A 3$ gene is composed of 1,670 amino acids, including a 28-amino acid signal peptide at the amino terminus, a carboxyl terminus containing 225 amino acids, and the important middle collagen area, which is rich in Gly (Gly-X-Y) triple-helix structure sequences (16). The intact triple-helix structure of type IV collagen is essential for GBM development and the maintenance of renal function, while a mutated triple-helix structure can induce a vicious cycle of abnormal signaling in the nephron and lead to marked extracellular matrix accumulation and glomerulosclerosis, causing progressive renal disorder (17). Since the molecular weight of Gly is the lowest amongst the amino acids, the three Gly residues can exactly match the inside part of the tightly folded triple-helix structure to maintain structural integrity $(16,18)$. Otherwise, if Gly is replaced by any other residue, an abnormally folded triple-helical collagen molecule would be highly sensitive to proteases and thus prone to degradation (18-20). Since the middle Gly (Gly-X-Y) triple-helix structure sequence is considerably long, gene mutations in different mutation sites would cause different clinical manifestations, largely depending on their role in the structural integrity $(16,18)$. In the present study, the proband was a patient with ADAS presenting with hematuria at only 5 years of age, at the time of IV collagen heterotrimer replacement (1), whereas the older sister exhibited overt symptoms considerably later. Therefore, the p.G868E mutation was pivotal in this case of ADAS. It was inferred that p.G195S was a less important conspirator, which did not cause abnormal clinical findings independently, but could synergistically accelerate disease progression. In particular, certain Gly substitutions in the triple-helix structure do not lead to pathological alterations and overt symptoms, further illustrating that the mutation site is critical in AS development and classification $(19,20)$.

Abnormal urinalysis and renal dysfunction are the important clinical manifestations of AS, and genetic screening is helpful for the diagnosis and prognosis of AS (21), as in the case of the pedigree in the present study. It was feasible to detect the pathogenic mutations of AS by targeted NGS or first-generation sequencing. However, there are three genes associated with AS, namely $C O L 4 A 3, C O L 4 A 4$ and COL4A5, which contain 52, 48 and 51 exons respectively (22). First-generation sequencing is very time-consuming and laborious and the results may be very frustrating, due to the pitfalls of sequencing large genes and the absence of known mutational hot spots $(21,22)$. At present, if the causative genes of inherited diseases are not clear, WES is a favorable alternative for identifying potentially causative variants with the advantages of time- and cost-efficiency (22-24). WES can provide detailed information on the sites of the inherited mutation to ensure timely medical intervention; if the proband and their family are diagnosed and administered ACEi earlier, renal function can be preserved for longer (6).

However, there were some limitations in the present study. Firstly, immunofluorescence examinations of all the family members of the pedigree were not performed due to the lack of resources and the patients' willingness. Secondly, the progress of the ADAS in the pedigree requires further follow-up to unveil the clinical characteristics and prognosis caused by these two mutations in the long-term. Finally, in order to thoroughly prove the effect of the two mutations' functional disadvantage, loss-of-function experiments for both in vivo and in vitro verification should be performed. In the future, the AS conditions of these siblings' future generations should be assessed, and any potential disease progression in the carriers of these two mutations should also be monitored if possible, to assist in timely medical interventions.

In conclusion, p.G868E is a vital mutation that leads to ADAS, whereas the p.G195S mutation can synergistically promote ADAS progression. These two mutations can provide novel insights into AS pathogenesis, and NGS may prove very effective in AS diagnosis and treatment. 


\section{Acknowledgements}

We would like to thank Professor Han Min (Department of Nephrology, Tongji Hospital, Tongji Medical College, Huazhong University of Science and Technology) for assistance with the electron microscopy analysis and pathological analysis of renal biopsies.

\section{Funding}

The present study was supported by the National Natural Science Foundation of China (grant no. 8200021897).

\section{Availability of data and materials}

All data generated or analyzed during this study are included in this published article. The novel COL3A4 mutation described in the present study has been submitted to ClinVar (accession no. VCV001177517.1; variant, NM_000091.5.).

\section{Authors' contributions}

$\mathrm{DN}$ and $\mathrm{CX}$ performed the experiments. DN and $\mathrm{ZZ}$ designed the study and drafted the manuscript. DN, KH and JL analyzed and interpreted the data. CW and TG collected the patient's data and followed up the case. All authors have read and approved the final manuscript. DN, CX and ZZ confirmed the authenticity of all raw data.

\section{Ethics approval and consent to participate}

The present study was approved by the Ethics Committee of Tongji Hospital, Tongji Medical College, Huazhong University of Science and Technology. Written informed consent for participation in the study was obtained from each patient, or from the parents of patients $<18$ years old.

\section{Patient consent for publication}

Written informed consent for publication of this study was obtained from each patient, or from the parents of patients $<18$ years old.

\section{Competing interests}

The authors declare that they have no competing interests.

\section{References}

1. Hudson BG, Tryggvason K, Sundaramoorthy M and Neilson EG Alport's syndrome, Goodpasture's syndrome, and type IV collagen. New Engl J Med 348: 2543-2556, 2003.

2. Kashtan C: Alport syndrome: Facts and opinions. F1000Res 6: $50,2017$.

3. Naylor RW, Morais M and Lennon R: Complexities of the glomerular basement membrane. Nat Rev Nephrol 17: 112-127, 2021.

4. Kruegel J, Rubel D and Gross O: Alport syndrome-insights from basic and clinical research. Nat Rev Nephrol 9: 170-178, 2013.

5. Crockett DK, Pont-Kingdon G, Gedge F, Sumner K, Seamons R and Lyon E: The Alport syndrome COL4A5 variant database. Hum Mutat 31: E1652-E1657, 2010.
6. Kashtan CE: Alport syndrome: Achieving early diagnosis and treatment. Am J Kidney Dis 77: 272-279, 2021.

7. Fallerini C, Dosa L, Tita R, Del Prete D, Feriozzi S, Gai G, Clementi M, Manna AL, Miglietti N, Mancini R, et al: Unbiased next generation sequencing analysis confirms the existence of autosomal dominant Alport syndrome in a relevant fraction of cases. Clin Genet 86: 252-257, 2014.

8. Omachi K and Miner JH: Alport syndrome therapeutics: Ready for prime-time players. Trends Pharmacol Sci 40: 803-806, 2019.

9. Xu Y, Lin Z, Tang C, Tang Y, Cai Y, Zhong H, Wang X, Zhang W, $\mathrm{XuC}$, Wang J, et al: A new massively parallel nanoball sequencing platform for whole exome research. BMC Bioinformatics 20: 153-161, 2019.

10. Huang J, Liang X, Xuan Y, Geng C, Li Y, Lu H, Qu S, Mei X, Chen $\mathrm{H}, \mathrm{Yu} \mathrm{T}$, et al: A reference human genome dataset of the BGISEQ-500 sequencer. Gigascience 6: 1-9, 2017.

11. Richards CS, Bale S, Bellissimo DB, Das S, Grody WW, Hegde MR, Lyon E and Ward BE: ACMG recommendations for standards for interpretation and reporting of sequence variations: Revisions 2007. Genet Med 10: 294-300, 2008.

12. Goka S, Copelovitch L and Levy Erez D: Long-term outcome among females with Alport syndrome from a single pediatric center. Pediatr Nephrol 36: 945-951 2021

13. Imafuku A, Nozu K, Sawa N, Hasegawa E, Hiramatsu R, Kawada M, Hoshino J, Tanaka K, Ishii Y, Takaichi K, et al: Autosomal dominant form of type IV collagen nephropathy exists among patients with hereditary nephritis difficult to diagnose clinicopathologically. Nephrology (Carlton) 23: 940-947, 2018.

14. Marcocci E, Uliana V, Bruttini M, Artuso R, Silengo MC, Zerial M, Bergesio F, Amoroso A, Savoldi S, Pennesi M, et al: Autosomal dominant Alport syndrome: Molecular analysis of the COL4A4 gene and clinical outcome. Nephrol Dial Transplant 24: 1464-1471, 2009.

15. Ninomiya Y, Kagawa M, Iyama K, Naito I, Kishiro Y, Seyer JM, Sugimoto M, Oohashi T and Sado Y: Differential expression of two basement membrane collagen genes, COL4A6 and COL4A5, demonstrated by immunofluorescence staining using peptide-specific monoclonal antibodies. J Cell Biol 130: 1219-1229, 1995.

16. Heidet L, Arrondel C, Forestier L, Cohen-Solal L, Mollet G, Gutierrez B, Stavrou C, Gubler MC and Antignac C: Structure of the human type IV collagen gene COL4A3 and mutations in autosomal Alport syndrome. J Am Soc Nephrol 12: 97-106, 2001.

17. Liu Y: New insights into epithelial-mesenchymal transition in kidney fibrosis. J Am Soc Nephrol 21: 212-222, 2010.

18. Yeo J, Qiu Y, Jung GS, Zhang YW, Buehler MJ and Kaplan DL: Adverse effects of Alport syndrome-related Gly missense mutations on collagen type IV: Insights from molecular simulations and experiments. Biomaterials 240: 119857, 2020.

19. Inoue $Y$, Nishio $H$, Shirakawa T, Nakanishi K, Nakamura $H$, Sumino K, Nishiyama K, Iijima K and Yoshikawa N: Detection of mutations in the COL4A5 gene in over 90\% of male patients with X-linked Alport's syndrome by RT-PCR and direct sequencing. Am J Kidney Dis 34: 854-862, 1999.

20. Gross O, Netzer KO, Lambrecht R, Seibold S and Weber M: Meta-analysis of genotype-phenotype correlation in X-linked Alport syndrome: Impact on clinical counselling. Nephrol Dial Transplant 17: 1218-1227, 2002.

21. Savige J, Ariani F, Mari F, Bruttini M, Renieri A, Gross O, Deltas C, Flinter F, Ding J, Gale DP, et al: Expert consensus guidelines for the genetic diagnosis of Alport syndrome. Pediatr Nephrol 34: 1175-1189, 2019.

22. Artuso R, Fallerini C, Dosa L, Scionti F, Clementi M, Garosi G, Massella L, Epistolato MC, Mancini R, Mari F, et al: Advances in Alport syndrome diagnosis using next-generation sequencing. Eur J Hum Genet 20: 50-57, 2012.

23. Morinière $\mathrm{V}$, Dahan $\mathrm{K}$, Hilbert $\mathrm{P}$, Lison $\mathrm{M}$, Lebbah $\mathrm{S}$, Topa $\mathrm{A}$, Bole-Feysot C, Pruvost S, Nitschke P, Plaisier E, et al: Improving mutation screening in familial hematuric nephropathies through next generation sequencing. J Am Soc Nephrol 25: 2740-2751, 2014 .

24. Tran KT, Le VS, Vu CD and Nguyen LT: A novel de novo variant of LAMA2 contributes to merosin deficient congenital muscular dystrophy type 1A: Case report. Biomed Rep 12: 46-50, 2020. 